\title{
Erratum: First-Principles Theory of Spatial Dispersion: Dynamical Quadrupoles and Flexoelectricity [Phys. Rev. X 9, 021050 (2019)]
}

Miquel Royo® and Massimiliano Stengel

(4) (Received 18 February 2022; published 8 March 2022)

DOI: 10.1103/PhysRevX.12.019903

After the publication of the article, we unfortunately discovered an incorrect negative sign in the right-hand side of Eq. (47b). This error also affects Eqs. (54) and (58). The corrected formulas are provided below.

$$
\begin{gathered}
i E_{\beta}^{\mathcal{E}_{\beta}^{*} \lambda}+i E_{\alpha}^{\mathcal{E}_{\beta}^{*} \lambda}=E_{\alpha \beta}^{\varphi^{*} \lambda}, \\
E_{\gamma \delta}^{\varphi^{*} \tau_{\kappa \beta}}=i E_{\gamma}^{\mathcal{E}_{\delta}^{*} \tau_{\kappa \beta}}+i E_{\delta}^{\mathcal{E}_{\gamma}^{*} \tau_{\kappa \beta}}, \\
P_{\delta, \kappa \beta}^{(1, \gamma)}=-\frac{2 i}{\Omega} E_{\gamma}^{\mathcal{E}_{\delta}^{*} \tau_{\kappa \beta}} .
\end{gathered}
$$

Published by the American Physical Society under the terms of the Creative Commons Attribution 4.0 International license. Further distribution of this work must maintain attribution to the author(s) and the published articles title, journal citation, and DOI. 